NASA/TM-2012-216050

\title{
Integrated RF/Optical Interplanetary Networking Preliminary Explorations and Empirical Results
}

Daniel E. Raible and Alan G. Hylton

Glenn Research Center, Cleveland, Ohio 


\section{NASA STI Program . . . in Profile}

Since its founding, NASA has been dedicated to the advancement of aeronautics and space science. The NASA Scientific and Technical Information (STI) program plays a key part in helping NASA maintain this important role.

The NASA STI Program operates under the auspices of the Agency Chief Information Officer. It collects, organizes, provides for archiving, and disseminates NASA's STI. The NASA STI program provides access to the NASA Aeronautics and Space Database and its public interface, the NASA Technical Reports Server, thus providing one of the largest collections of aeronautical and space science STI in the world. Results are published in both non-NASA channels and by NASA in the NASA STI Report Series, which includes the following report types:

- TECHNICAL PUBLICATION. Reports of completed research or a major significant phase of research that present the results of NASA programs and include extensive data or theoretical analysis. Includes compilations of significant scientific and technical data and information deemed to be of continuing reference value. NASA counterpart of peer-reviewed formal professional papers but has less stringent limitations on manuscript length and extent of graphic presentations.

- TECHNICAL MEMORANDUM. Scientific and technical findings that are preliminary or of specialized interest, e.g., quick release reports, working papers, and bibliographies that contain minimal annotation. Does not contain extensive analysis.

- CONTRACTOR REPORT. Scientific and technical findings by NASA-sponsored contractors and grantees.
- CONFERENCE PUBLICATION. Collected papers from scientific and technical conferences, symposia, seminars, or other meetings sponsored or cosponsored by NASA.

- SPECIAL PUBLICATION. Scientific, technical, or historical information from NASA programs, projects, and missions, often concerned with subjects having substantial public interest.

- TECHNICAL TRANSLATION. Englishlanguage translations of foreign scientific and technical material pertinent to NASA's mission.

Specialized services also include creating custom thesauri, building customized databases, organizing and publishing research results.

For more information about the NASA STI program, see the following:

- Access the NASA STI program home page at http://www.sti.nasa.gov

- E-mail your question to help@sti.nasa.gov

- Fax your question to the NASA STI Information Desk at 443-757-5803

- Phone the NASA STI Information Desk at 443-757-5802

- Write to: STI Information Desk NASA Center for AeroSpace Information 7115 Standard Drive Hanover, MD 21076-1320 
NASA/TM-2012-216050

\section{Integrated RF/Optical Interplanetary Networking Preliminary Explorations and Empirical Results}

Daniel E. Raible and Alan G. Hylton

Glenn Research Center, Cleveland, Ohio

National Aeronautics and

Space Administration

Glenn Research Center

Cleveland, Ohio 44135 


\section{Acknowledgments}

The authors would like to thank the NASA Space Communication and Navigation (SCaN) program, and in particular Mr. John Rush, for supporting this research.

Level of Review: This material has been technically reviewed by technical management.

Available from

NASA Center for Aerospace Information 7115 Standard Drive

Hanover, MD 21076-1320
National Technical Information Service 5301 Shawnee Road Alexandria, VA 22312 


\title{
Integrated RF/Optical Interplanetary Networking Preliminary Explorations and Empirical Results
}

\author{
Daniel E. Raible and Alan G. Hylton \\ National Aeronautics and Space Administration \\ Glenn Research Center \\ Cleveland, Ohio 44135
}

\begin{abstract}
Over the last decade interplanetary telecommunication capabilities have been significantly expanded-specifically in support of the Mars exploration rover and lander missions. NASA is continuing to drive advances in new, high payoff optical communications technologies to enhance the network to Gbps performance from Mars, and the transition from technology demonstration to operational system is examined through a hybrid RF/optical approach. Such a system combines the best features of RF and optical communications considering availability and performance to realize a dual band trunk line operating within characteristic constraints. Disconnection due to planetary obscuration and solar conjunction, link delays, timing, ground terminal mission congestion and scheduling policy along with space and atmospheric weather disruptions all imply the need for network protocol solutions to ultimately manage the physical layer in a transparent manner to the end user. Delay Tolerant Networking (DTN) is an approach under evaluation which addresses these challenges. A multi-hop multi-path hybrid RF and optical test bed has been constructed to emulate the integrated deep space network and to support protocol and hardware refinement. Initial experimental results characterize several of these challenges and evaluate the effectiveness of DTN as a solution to mitigate them.
\end{abstract}

\section{Introduction}

Over the last decade interplanetary telecommunication capabilities have been significantly expanded, and specifically in support of the Mars exploration rovers Spirit (MER-A) and Opportunity (MER-B), Phoenix Lander and orbital missions such as Mars Express, Mars Odyssey, and Mars Reconnaissance Orbiter. This trend will continue with the NASA Curiosity rover, the planned MAVEN orbiter, ESA ExoMars rover, potential sample return (Ref. 1) and/or human exploration precursor missions (Ref. 2) under the Mars Next Generation Program Planning Group. Orbital relays have provided increased energy efficiency per bit and greater volume of science data return from resource constrained surface assets while concurrently supporting the onboard orbital science objectives. This relay approach will continue into the next decade of Mars exploration which may include joint NASA/ESA orbiters and rovers along with architecture developments to enable future sample return and deep space human exploration initiatives. Such activities will demand a communications availability and quality of service far beyond what has been deployed thus far in order to monitor critical events such as precision rendezvous and docking, distribute timing information, supplement navigational services and in general utilize the exploration vehicles and science spacecraft operating on or near the Mars surface to their fullest potential.

The NASA Space Communication and Navigation (SCaN) program's integrated network development plan places an emphasis on interoperability to facilitate compatibility between missions and with other space agencies which will influence the requirements levied on deep space relays to support multiple and evolving operations. A notional example of the communications architecture is shown in Figure 1, which illustrates the continued use of relay communications payloads on science orbiters along with dedicated communication relay satellites with store-and-forward and space internetworking capabilities. 


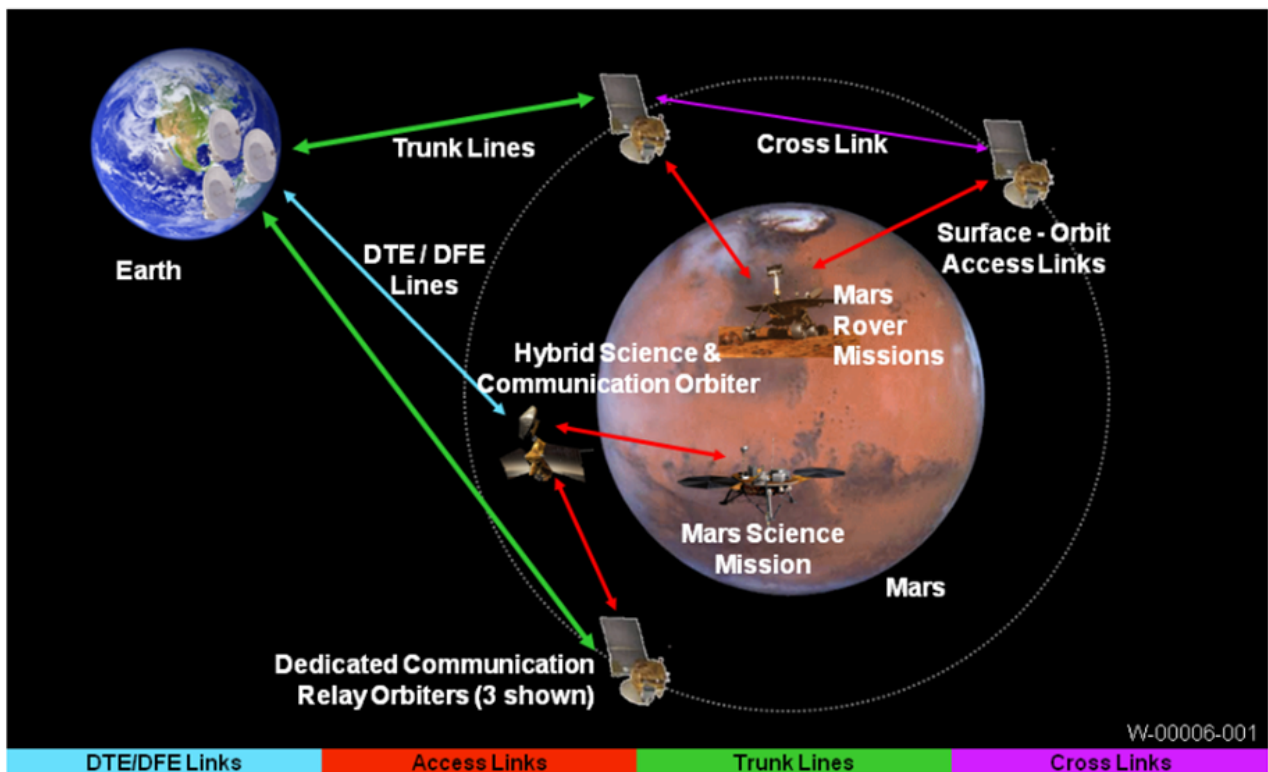

Figure 1.-Mars relay capability (Ref. 3).

The future Mars robotic science and human exploration precursor missions will require enhancements to the existing telecommunications infrastructure to include extended space internetworking services for each of the nodes, and integrated network management to operate the assets as essential elements of the SCaN service framework. Furthermore the infusion of optical communications technologies into the network promises a substantial enhancement in link performance with higher data rates unbounded by spectrum regulations. Optical communications offers secondary benefits in the form of lower transceiver size, mass and prime power requirements. The component technologies necessary to realize such a system are available now, and the overarching challenge is how to introduce the capability into a deep space network while minimizing spacecraft resources devoted to communications.

These areas of advancement are investigated at the NASA Glenn Research Center (GRC) under the Delay/Disruption/Disconnection Tolerant Networking (DTN) and Integrated Radio and Optical Communications (iROC) projects. The potential capabilities and infusion methodologies of DTN and iROC into the operational deep space network are being examined on the hardware and software sides of a flight communications payload. A multi-hop multi-path hybrid RF and optical test bed has been constructed to emulate the integrated deep space network and to support protocol and hardware refinement. Initial experimental results characterize several of these challenges and evaluate the effectiveness of DTN as a solution to mitigate them.

\section{RF/Optical System Description}

One of SCaN's primary goals is to develop a communications infrastructure that provides the highest data rates feasible so as to not limit the scientific capability of missions. While the exact communications system performance necessary to accomplish the future Mars missions is yet to be decided, it is clear that higher dates rates must be attained to sustain multiple synthetic aperture radar (SAR) and hyperspectral imaging instruments along with the demands of supporting a future human exploration initiative.

NASA is driving advances in new, high payoff optical communications technologies to meet this challenge and several technology demonstration missions are scheduled over the next few years. The Jet Propulsion Laboratory's (JPL) Deep Space Optical Terminal (DOT) project has consisted of a focused technology development effort that has matured many key technologies including pulse-position modulation (PPM) schemes, high-bandwidth control loops for fine beam pointing, stabilization platforms, precision ranging and radiation-hard leaser transmitters (Ref. 4). Much of this enabling technology has 
been carried over and further refined from the Mars Laser Communications Demonstration (MLCD) (Ref. 5) project, and these advancements serve to broaden the trade space of available tools to construct deep space optical communication systems. The DOT effort will go a long way toward mitigating the perceived risks of optical communications in a deep space environment and the continual development of the critical enabling technologies will further extend the feasible performance of such systems to realize $\mathrm{Mb} / \mathrm{s}$ data rates from Jupiter and Uranus (Ref. 6). NASA's Lunar Laser Communications Demonstration (LLCD) is also making a similar technology push and has brought along the advancements with superconducting photon counting detectors and high peak-power doped-fiber transmitters (Ref. 7).

Other critical technologies toward making successful high speed optical communications systems include the development of atomic clocks and oscillators capably of exceeding $\sim 10^{-13}$ stability (Ref. 8). Clock distribution and synchronization between deployed assets is crucial for navigation, radiometric science, beam pointing control and data synchronization between network nodes. The onboard clocks systems must be able to periodically correct for relativistic time dilations due to gravitational redshift, and be robust enough to handle the ionizing radiation characteristic of the space environment. The ability to maintain accurate synchronization between each of the network assets is crucial towards enhancing the art of deep space operations and harnessing the full potential of the enabling physical layer technologies.

The evolution of optical communications technology will enhance the SCaN network to Gbps performance from Mars, and the transition from technology demonstration to operational system is examined through a hybrid RF/optical approach (Ref. 9). The iROC project (Fig. 2) is taking this approach, in an effort to accelerate the infusion of optical communication technologies into the network timeline through realizing that both RF and optical systems must coexist on the spacecraft for some period of time at a minimal size, mass and power penalty to the mission. Such a system combines the best features of RF and optical communications considering availability and performance to realize a dual band trunk line operating within characteristic constraints of current infrastructure and projected development resources. The iROC approach takes advantage of integration strategies primarily through a combined RF/optical aperture, shared gimbal, integrated high bit rate digital core encoders/modulators, duty cycle scheduling of prime power and sharing of the supporting structural and thermal elements. Through the leveraging of previously mentioned technologies under the DOT and LLCD projects, iROC link budget analysis suggests feasible data rates in the hundreds of $\mathrm{Mb} / \mathrm{s}$ for both the RF and optical connections direct to Earth (DTE) from a Mars orbiter.

Harnessing the link capability through a networking architecture will be essential toward utilizing the deployed communications systems in an efficient manner to maximize overall throughput throughout a lifecycle that may see evolutions from robotic missions. Disconnection due to planetary obscuration and solar conjunction, link delays, timing, ground terminal mission congestion and scheduling policy along with space and atmospheric weather disruptions all imply the need for network protocol solutions to ultimately manage the physical layer in a transparent manner to the end user. Furthermore the data rate, quality of service and security needs for future deep space robotic, sample return and human exploration missions will be different, and the deployed integrated network solution must be general enough to adapt over time to address these demands.

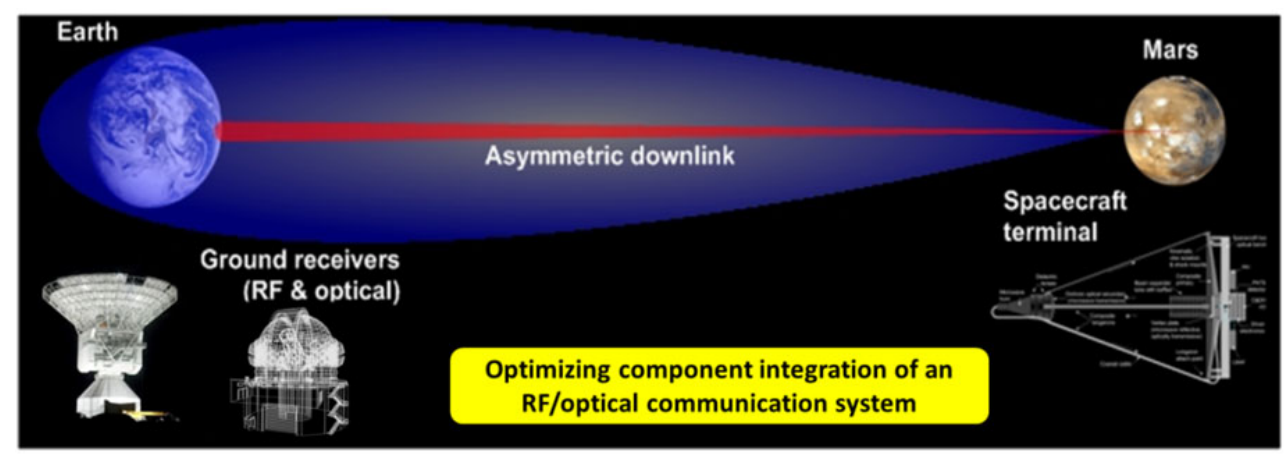

Figure 2.-Dual-band deep space communications trunk line (Image courtesy APL). 
Future communications assets will need to be implemented in compliance with Consultative Committee for Space Data Systems (CCSDS) standards and Interagency Operations Advisory Group (IOAG) space internetworking recommendations to maximize interoperability between partner agencies, and to maximize commonality in network and service management. Delay/Disruption/Disconnection Tolerant Networking (DTN) is an approach under evaluation which addresses these challenges. DTN may also enable seamless infusion of integrated RF/optical links into existing and future networks. Ultimately it is the role of DTN to make the minutia and idiosyncrasies of the integrated network appear as transparent as possible to the end user, while maintaining a quality of service appropriate for each traffic type.

An examination of the integrated approach has been made for even larger distances out to 1000 AU with the Realistic InterStellar Explorer (RISE) (Ref. 10) advanced concept. In the RISE design, the considerable range prohibits interactive control of the spacecraft from Earth, necessitating autonomous pointing operation based on celestial navigation. The optical link is used primarily for data return, and the RF system is used for command uplink. The novel integration of the RISE design offers a compact and power efficient payload, but will require higher wall-plug efficient and lightweight components to be developed.

In the private sector side, Planetary Resources is designing spacecraft for water and precious mining of asteroids and their designs are based around a core integrated optical communications technology which would provide image capturing, navigation duties through star tracking and laser-based communication to Earth all through the same instrument (Ref. 11). The spirit of this integration philosophy may be traced back to the NASA Lunar Reconnaissance Orbiter's Mini-RF payload which provided both SAR along with S and X-band communications capability from a single instrument. Moving toward the future, customers wanting increased functionality from smaller payloads will drive further architecture integrations considering reconfigurability and reusability of spacecraft elements during different operational states. Such an evolution will challenge the classical spacecraft architecture definitions of mapping a single function to a single subsystem, and will ultimately achieve more operational duty cycle from the elements as they contribute more towards the overall functionality of the spacecraft.

Finally, free space hybrid RF/optical networking has been closely examined on board airborne platforms through the DARPA Optical RF Communications Adjunct (ORCA) program and its follow-on, Free-space Optical Experimental Network Experiment (FOENEX) program (Ref. 12). ORCA/FOENEX took advantage of layer 2 retransmission triggers to quickly recover lost data. This method has been used successfully to delivery data at a high 99 percent reliability with minimal impacts on latency. It is important to note that due to the relatively close proximity of the nodes in ORCA/FOENEX, hybrid link failover from one disrupted link to another functional one was possible through the hybrid router, but a scheme such as this would be difficult to implement in a deep space system due to the large link delay and thus feedback latency.

\section{Connectivity Analysis}

General concept of operation guidelines for near Earth and deep space scenarios may be found in Reference 13, and these may be tailored to meet specific mission criteria. Perhaps one of the most constrained missions would be the combined orbital science and data relay orbiter. These missions nominally serve a 2 year science objective, followed by a 3 year relay objective. Since the asset is placed in a suboptimal orbit from a telecommunications perspective (so as to facilitate the necessary imaging resolutions), the connectivity with Earth is very sporadic due to planetary obscuration. Furthermore, the orbiter may need to have a complimentary operational duty cycle between the scientific instrumentation and the communications system due to pointing and trajectory limitations created by gimbal constraints.

In considering deep space scenarios, the important characteristics of large and varying range distances and varying geometric angles between the Sun, Earth and spacecraft drive the variable performance and scheduled connections of the communication links over a considered mission lifetime. Each connection would initialize with the acquisition sequence, after which data would be transferred utilizing an underlying synchronization format, and ending with either an orderly termination of the link or an unscheduled interruption that must be handled to reestablish the data transfer. 
For the deep space hybrid RF/optical link to be successful it needs to be engineered as an integral part of a larger system. In other works, if the network is assembled in a piecewise fashion from each of its individual links, it will be lacking the autonomy to adapt in an efficient manner to the operational environment and evolving demands from the users as new missions are introduced. On the software side iROC is a high bit rate link, although the RF and optical links will have widely disparate rates at times. The conditions under which iROC nodes operate present a variety of challenges that must be overcome to be rendered useful. Firstly, there is disconnection due to scheduled ground station handoffs which disrupt connectivity, unplanned obscuration of the optical beam from atmospheric effects, logistics issues with from the Laser Clearing House and FAA if an uplink beacon is used, deep atmospheric fading and competition with other assets considering resource scheduling and management. Secondly, the round trip light times will at times exceed $30 \mathrm{~min}$ and this high latency makes it difficult to get receipts. iROC networks will need buffering to handle the disconnections, routing to move data across a multi-hop multi-path store and forward system and security to protect from the inside as well as the outside of the network.

There have been many studies made to determine the optimal number and location of optical ground terminals around the globe, but to move forward with deploying a deep space communications package a significant performance metric must be attainable with a minimum of infrastructure. Investigations have been made into the utilization of a single optical site (SOS) in order to work within practical resource constraints, and this has proven to be a cost effective way to initially establish a capable operational system that can expand over time (Ref. 14). The SOS approach will place a demand on the amount of spacecraft memory required for buffering, but this cost is negligible compared with the construction of global terrestrial optical ground terminal construction.

Several Mars architectures have been recommended, and the direction that the current system takes is dependent on many factors ranging from political to financial to technical. In general, the topology would consist of multiple surface assets talking back to multiple Deep Space Network (DSN) sites at Earth through multiple Mars Telecommunications Orbiters (MTO). Figure 3 is a depiction of such a system, with the addition of an Earth Telecommunications Orbiter (ETO). This is a useful starting point to conduct analysis, as it represents a multi-node multi-hop system that may be easily scaled to represent similar and evolving architectures.

One unique characteristic of an integrated RF/optical payload is a co-boresighted aperture, which may allow simultaneous transmission on both the RF and optical channels. A concept of operations for such an arrangement may take advantage of the spatial proximity of the Goldstone DSN complex with the planned optical ground terminal. Since the RF beamwidth of a typical antenna transmitting from Mars is multiple times the diameter of the Earth, it can be realized that anytime the optical link is active there may be a simultaneous RF link established with Goldstone. This presents an opportunity for the network to operate in a multicast mode, which is a way of addressing multiple nodes at once. As the ground network expands these opportunities will increase, and the network should be flexible enough to accommodate them.

Satellite Tool Kit (STK) may be used to determine the connectivity of a combined RF/optical Mars mission. Using the MRO orbit as a baseline for comparison and the SOS concept, the total daily contact times between the DSN sites and the optical site may vary between 10 to $20 \mathrm{hr}$ depending on the time of year. These values represent the reductions due to elevation restrictions, scheduling and weather masking at the ground receivers, and in general the total daily contact times are realized from many passes lasting

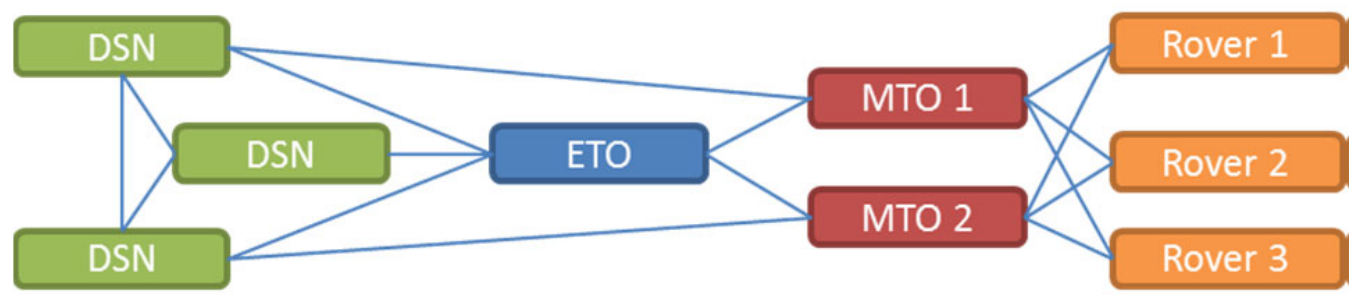

Figure 3.-Potential Mars-Earth network topology. 
anywhere from 40 to $70 \mathrm{~min}$. The considerable amount of daily contacts imply many disconnections and handoffs that will have to be handled at the network layer to minimize the burden on the spacecraft storage through intelligent retransmissions when necessary. The scheduling output from the STK simulations will be used to conduct the subsequent experimental emulations on the optical test bed.

\section{Experimental Setup}

Previous work at JPL has reported on an initial experiment characterizing the performance of a DTN enabled point-to-point space optical link, and through this it was learned that excellent system performance can be achieved on a disconnected link by using link-state information to burst high rates of data upon reacquisition (Ref. 15). The experimental setup picks up where the point-to-point link left off. DTN adds the capability needed to think about iROC in bigger terms than a simple point-to-point link, and this is realized through a multi-node multi-hop test bed to serve as a research platform for new DTN software components and provide honest network characterization for present and future missions.

The initial test bed consists of five network nodes connected by six free space optical links. One end of the test bed reaches out to the Mars surface robot to receive data to be relayed through the network and channel emulator, and the other side of the test bed delivers the routed data to the Mission Operations Center (MOC) back at Earth (Fig. 4). The simulation results from STK are used to drive the network, so the experiments are conducted in a manner that emulates realistic mission contacts and disconnections.

Each of the computer nodes in the network are connected through a bidirectional optical Ethernet convertor, which is launched into a free space optic system on the optics bench (Fig. 5).

The test bed is modeled after a scenario in which a Mars orbiter sends data to a MOC. The orbiter sends data direct-to-earth (DTE) and has contact times scheduled with three different deep space network (DSN) sites. The DSN sites are also networked to the MOC. This is a multi-hop multi-path network.

To generate realistic connectivity times and link delays, the scenario was laid out in the STK software package. A Mars orbiter was placed along with three DSN sites (Canberra, Goldstone, and Madrid). The data, summarized in Azimuth, Elevation, and Range (AER) reports from which the DTN configurations were distilled. The selected time period was over the month of June in 2012.

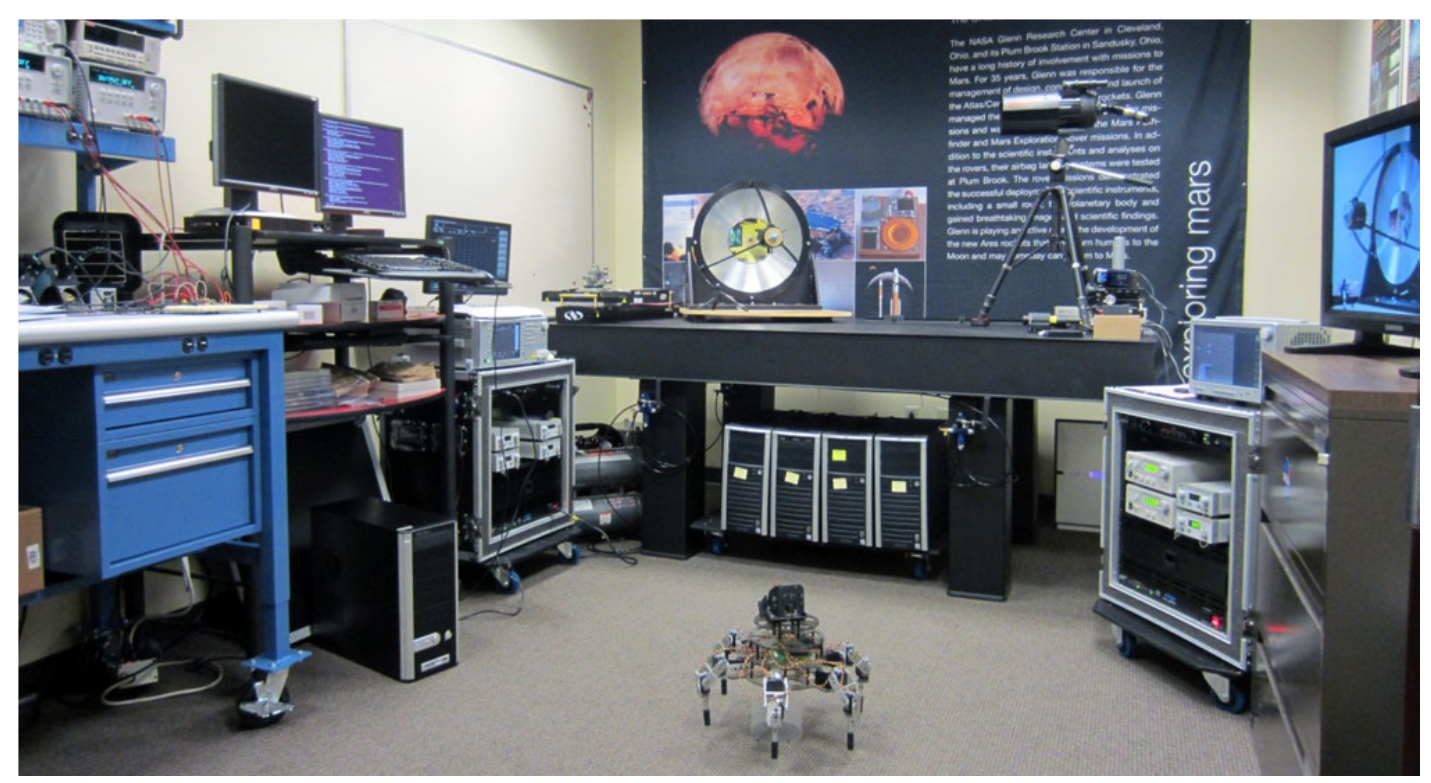

Figure 4.-Multi-node multi-hop free space optical DTN test bed. 

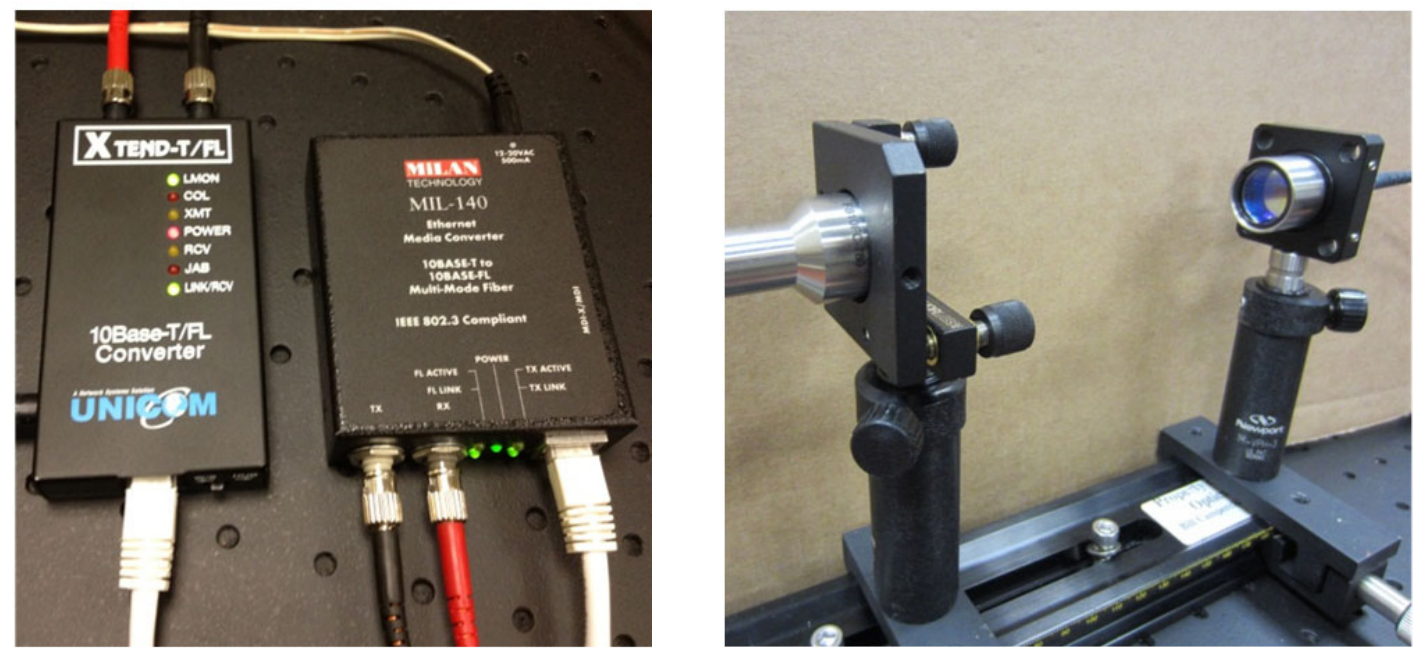

Figure 5.-Optical ethemet convertors and free space optics.

There are six computers in total. Five of the computers are the DTN nodes (The orbiter, the MOC, and the three DSN sites). The sixth computer is a channel emulator that reproduces the Mars to Earth link characteristics - notably the delay. During the chosen test period, Mars was on average 10 min away by light (20 min round trip time). There are multiple implementations of DTN, and ION version 3.0.1 was selected. ION, developed by JPL and NASA, computes routes based on known contact times. This is valid in the network described, as the assets' connectivity are all determined by orbital mechanics (Mars to Earth) or are constant (DSN sites to the MOC). The channel emulator is an open software package developed at the NASA Glenn Research Center for earlier projects.

The CPUs in the computers are all first generation Pentium 4s, and each computer has $512 \mathrm{MB}$ of RAM. As satellites are limited platforms by terrestrial standards, it would not be fair to have modern machines. Each computer runs Ubuntu 11.10. Due to the 10 min link delay, the Address Resolution Protocol (ARP) had to be set statically. Additionally, significant IPv6 traffic was observed over the links with 10 min delays and therefore IPv6 was disabled on all machines. The primary unit of data in DTN, the bundle, has a timeout based on seconds. Furthermore, connectivity is time based, so all computers had to be synchronized. Clock drift was observed even in the lab environment, so an Ethernet backchannel was present to all nodes so that one could act as a Network Time Protocol (NTP) server to achieve synchronization.

\section{Preliminary Results}

The basic actions of the generic DTN are to store, carry, and forward data. All networks forward data towards the recipient or recipients. The storage of large amounts of data for prolonged amounts of time and the potential mobility required to carry data to the next forwarding agent (also known as a hop) set DTN apart from traditional networking concepts. Each of these simple words runs deeply with both known and unknown implications that are being uncovered and resolved with modern DTN research.

Storing data enables a disconnected node to wait for a contact period with a neighbor. During disconnection it is likely that additional data will be added to the buffer. This only partially determines local buffer requirements; indeed, if a node has to store its own data and a neighbor's, then there will be contention for limited resources. The notion of policies to ensure gracefulness is an ongoing area of research. Another capability added by carrying data is reliability - if the data is held even after transmission, then retransmissions are straightforward. Perhaps a more subtle difficulty overcome with storage is that of link asymmetry. If a node receives data quicker than it can send it, the data must be buffered or lost. DTNs handle this problem automatically; however this requires careful planning for storage requirements. 
Carrying data allows nodes to either wait for a neighbor to arrive or to move and go to a neighbor. As a node moves, perhaps in orbit, it may receive data from neighbors. As described, this might complicate matters from a storage standpoint as the policies local to the orbiting node cannot be synchronized with nodes passed. As connectivity might not be possible (no line of sight) or link delays get too large, feedback loops are not possible. Another difficulty encountered with the carrying of data is that addressing is not well-defined in DTN. Addressing in a network tells where a node is so that when forwarding data a route can be chosen so that with each transmission the data moves closer to its destination. Without an addressing structure this information must be determined by other means. In our case, this is possible as all connection periods can be calculated. This is not possible with all nodes in a more general network.

When testing ION in our network, the ultimate goal is to get as much data from Mars to the Earth as possible. This implies reliability on ION's part as well as efficient link utilization. Every time a bundle is sourced from the Mars orbiter node, new information has to be added to the bundle queue within ION. This queue will be at its largest when many outgoing bundles are added during periods of complete disconnection, during which time the behavior of ION will come under particular scrutiny.

Part of testing protocols is understanding their breaking points, and we uncovered one such point when sending bundles larger than $64 \mathrm{MB}$ in our configuration. Therefore, the largest bundles sent were $64 \mathrm{MB}$. We chose to send one $64 \mathrm{MB}$ bundle and then one random bundle (10 k, $100 \mathrm{k}, 1 \mathrm{M}$, or $10 \mathrm{M})$ and repeat every three seconds. The original intention was to run two tests-one with custody transfer and one without. Custody transfer is the aforementioned mechanism by which retransmission may occur if necessary. On a spacecraft, all processes are granted a fixed amount of memory. What was originally computed to be sufficient for ION turned out to be incorrect, and after a while new bundles were immediately dropped by the Mars node.

The memory was thus increased as far as ION would allow without crashing. It turned out that the additional memory was also insufficient, but during a different period. The first test, with the smaller memory pool, ran out of memory during a contact period. The first test was rerun with more memory which ran out of memory during a period of total disconnection. The test with custody transfer was also run with the larger amount of memory, which ran out of memory at the same point as the first repeated test.

This result actually makes a lot of sense. If larger bundles could be sourced, then fewer entries would be added to the queue. This would prevent overloading the protocol and present less of a burden to the processor and memory, but more of a burden to the non-volatile storage (hard drive or solid state drive). The periods of disconnection lasted on the order of hours, and memory ran out after 2700 additional bundles were sourced which is fair. This problem was amplified by the fact that the transmission from Mars to the DSN sites ran much slower than line rate and therefore the three second delay between transmissions was insufficient. It was found that with custody transfer nearly half of the received bundles were $64 \mathrm{MB}$, which reflects the sender's situation. Without custody transfer more bundles were received but less than 35 percent of the received bundles were $64 \mathrm{MB}$.

To avoid having thousands of bundles in the queue there are several possible mechanisms. One is to extend the protocol to send larger bundles, which in simpler circumstances it already can. Additionally, it may be useful to aggregate smaller bundles into larger bundles. For example, during a period of disconnection, ION might try to assemble bundles that are at least $512 \mathrm{MB}$. This would resemble the way Amazon ships books to consumers-not one book per box. Note that this approach is not entirely custody transfer friendly. Reliability in the form of retransmissions would become more expensive. It may be that not every piece of an aggregate bundle needs to be retransmitted, but to send pieces the custody signal would have to be at least somewhat content aware to avoid undue link congestion and at any rate the entire aggregate would have to be stored on the space craft. This and other considerations on the order of fragmentation merit further study. 


\section{Conclusions and Future Work}

The initial test bed to support hybrid RF/optical deep space investigations for iROC and DTN has been successfully constructed and operated. Initial implications over memory allocation and custody transfer have been highlighted in the mission scenario, and there now exists a capability to research further into these and subsequent issues. Over time it is planned to expand the test bed to conduct complete network experiments featuring RF, optical and wired (ground) links, which will allow further research into multicast and dynamic routing capabilities. More relay assets will be added, including the UHF links typical of resource constrained surface instruments. Physical layer channel emulations of atmospheric fades, background noise, unscheduled disconnections and pointing losses are also in the planning stages to evaluate the robustness of the network.

Further DTN testing will explore security issues and policy management, along with developing requirements for on board memory, software footprint and power requirements. Preparations are currently underway to expand the link range of the test bed by replacing the Ethernet to fiber convertors with dedicated $1550 \mathrm{~nm}$ lasers and electro optic modulators, which are launched into telescopes to place the detectors as far away as $1500 \mathrm{~m}$ (Fig. 6). This new arrangement will allow a portion of the test bed to be operated in actual weather conditions, thus adding to the unpredictable and honest evaluation of the network.

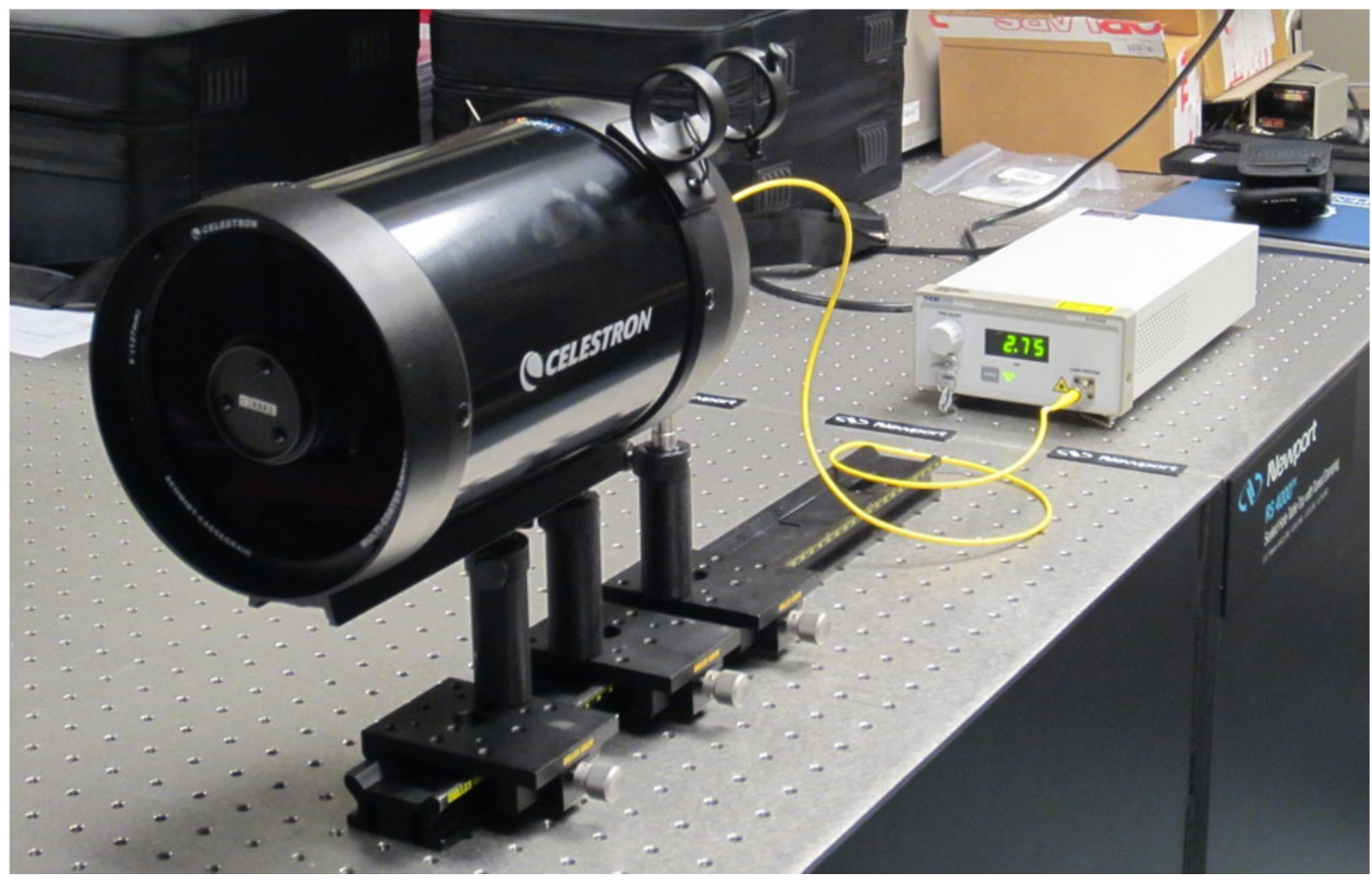

Figure 6.-Launching fiber-coupled laser into telescopes. 


\section{References}

1. “Mars Sample Return,” European Space Agency, December 8, 2009, Retrieved 2012-07-25. http://exploration.esa.int/science-e/www/object/index.cfm?fobjectid=44995

2. "NASA Aims to Put a Man on Mars by 2037," Mars Daily, September 24, 2007, Retrieved 2012-0725. http://www.marsdaily.com/reports/NASA_aims_to_put_man_on_Mars_by_2037_999.html

3. Younes, B., Perko, K., Shier, J., "Space Communications and Navigation (ScaN) Network Architecture Definition Document (ADD) Volume 1: Executive Summary,” NASA SCaN-ADD-V1, Revision 2, October 31, 2011, p. 21.

4. Biswas, A., Hemmati, H., Moison, B., “Deep-space Optical Terminals (DOT) System Engineering,” IPN Progress Report 42-183, November 15, 2010.

5. Boroson, D.D., Biswas, A., Edwards, B.L., "MLCD: Overview of NASA’s Mars Laser Communications Demonstration System,” LEOS Summer Topical Meetings, 2005 Digest of the, vol., no., pp. 5-7, 25-27 July 2005.

6. Hemmati, H., Biswas, A., Boroson, D.M., "Prospects for Improvement of Interplanetary Laser Communication Data Rates by 30 dB,” Proceedings of the IEEE, vol. 95, no. 10, pp. 2082-2092, Oct. 2007.

7. Robinson, B.S., Boroson, D.M., Burianek, D.A., Murphy, D.V., “The Lunar Laser Communications Demonstration,” Space Optical Systems and Applications (ICSOS), 2011 International Conference on , vol., no., pp. 54-57, 11-13 May 2011.

8. Prestage, J.D., Weaver, G.L., “Atomic Clocks and Oscillators for Deep-Space Navigation and Radio Science,” Proceedings of the IEEE, vol. 95, no. 11, pp. 2235-2247, Nov. 2007.

9. Fielhauer, K.B., Boone, B.G., Raible, D.E., "Concurrent System Engineering and Risk Reduction for Dual-Band (RF/optical) Spacecraft Communications,” Aerospace Conference, 2012 IEEE, vol., no., pp.1-7, 3-10 March 2012 doi: 10.1109/AERO.2012.6187116.

10. Bradley G. Boone; Robert S. Bokulic; G.B. Andrews; Ralph L. McNutt, Jr.; Nicholas G. Dagalakis, "Optical and Microwave Communications System Conceptual Design for a Realistic Interstellar Probe,” SPIE Proceedings Vol. 4821, 9 December 2002, pp. 225-746.

11. Leone, D., "Planetary Resources Seeks NASA Funds for Optical Technology,” Space News, Vol. 23, Issue 20, May 21, 2012, p. 14.

12. Zachary C. Bagley, David H. Hughes, Juan C. Juarez, Paul Kolodzy, Todd Martin, Malcolm Northcott, H. Alan Pike, Ned D. Plasson, Brian Stadler, Larry B. Stotts and David W. Young, “Hybrid Optical Radio Frequency Airborne Communications,” Opt. Eng. 51, 055006 (May 25, 2012).

13. Schultz, K.S., Rush, J., “Optical Link Study Group Interim Report,” Interagency Operations Advisory Group, December 1, 2011.

14. Noreen, G., Shambayati, S., Piazzolla, S., Cesarone, R., Strauss, K., Amoozegar, F., "Low Cost Deep Space Hybrid Optical/RF Communications Architecture,” Aerospace conference, 2009 IEEE, 7-14 March 2009.

15. Schoolcraft, J., Wilson, K., "Experimental Characterization of Space Optical Communications With Disruption-Tolerant Network Protocols,” Space Optical Systems and Applications (ICSOS), 2011 International Conference on, 11-13 May 2011. 

